\title{
Mineração
}

\section{Garantia financeira para fins de fechamento de mina e o seu impacto na viabilidade de uma mina. Estudo de caso de uma mina de grande porte}

\author{
(Financial guarantee for mine closure and its impact on the \\ feasibility of a mine. The study of case of a large mine)
}

\author{
Maurício Rios de Almeida \\ Mestre em Engenharia Civil. Gerente de Contratos da CONCREMAT S.A. \\ E-mail:mrios@uai.com.br
}

Hernani Mota de Lima

Professor Adjunto do Departamento de Engenharia de Minas/Escola de Minas/UFOP

E-mail:hernani.lima@ufop.br

\begin{abstract}
Resumo
A viabilidade da explotação dos bens minerais deve ser avaliada por seu custo de oportunidade. A mineração, quando praticada de forma técnica, ambiental e socialmente satisfatória, gera renda, empregos e benefícios a toda sociedade. Embora as minerações devam apresentar e implementar um PRAD para minas ao fim da fase produtiva, não existe, no Brasil, um sistema regulatório para tratar das questões relativas ao fechamento de mina e, particularmente, para o caso da garantia financeira que assegure, ao final da vida útil da mina, a disponibilidade de recursos necessários ao fechamento desta em caso de falta do minerador. Garantia financeira para fechamento de mina é, simplesmente, uma garantia de que o plano de fechamento será implementado. É um instrumento para proteger a sociedade dos passivos ambientais de um fechamento de mina. Esse artigo avalia o impacto da aplicação da garantia financeira na viabilidade de uma mina de grande porte no Estado de Minas Gerais.
\end{abstract}

Palavras-chave: Economia mineral, fechamento de mina, garantia financeira, política mineral, viabilidade.

\begin{abstract}
A mineral resource exploitation's feasibility should be evaluated by its opportunity cost. When properly practiced, technically, environmentally and socially, mining generates income, employment and benefits to society. Although mining companies are required to present and implement a PRAD (a mine closure plan), Brazil lacks a regulatory system to address mine closure issues; in particular, financial guarantee to assure that the necessary resources for closure will be available at the end of the mine's life cycle, regardless of the operator's financial position at closure. Financial guarantee for mine closure and post closure is simply a guarantee that the closure plan will be implemented. Financial guarantee is designed to protect the community from environmental liabilities at mine closure. This paper evaluates the impact of financial guarantee in the feasibility of a large mine operation in Minas Gerais State.
\end{abstract}

Keywords: Mineral economy, mine closure, financial guarantee, mineral politics, feasibility. 


\section{Introdução}

Em diversos países, companhias de mineração têm sido requeridas a depositar, no órgão governamental, uma caução como forma de garantia de implementação das atividades de fechamento e reabilitação de uma mina. Diversos são os intrumentos de garantia financeira para fins de fechamento de mina. Os intrumentos mais comuns incluem depósitos em bancos, bônus de desempenho, carta de crédito, fundos de investimento, propriedades \& ativos, certificados de depósito e seguros (Miller, 2005 e Bamburra, 2003).

Miller (2005) apresenta os vários mecanismos de garantia financeira exigida em alguns países:

- Austrália - New South Wales: cash e bank guarantee.

- Austrália - Northern Territory: cash e unconditional bank guarantee.

- Austrália - Queensland: cash, bank guarantee (unconditional, irrevocable, on demand); insurance bond (unconditional, irrevocable, etc).

- Austrália - South Australia: cash, irrevocable bank guarantee or letter of credit.

- Austrália - Tasmânia: cash deposit, bank guarantee, term deposit ou outra forma determinada pelas autoridades locais.

- Austrália: Victoria: cash, irrevocable bank guarantee ou letter of credit.

- Australia - Western Australia: unconditional guarantee de uma instituição financeira bancária.

- Canadá - Ontario: cash, letter of credit, bond aprovado por uma seguradora, trust, corporate financial test, ou outras formas aprovadas pelas autoridades locais.

- Canadá - Quebec: cheque, bond issue or guarantee investment certificates, term deposit, irrevocable e unconditional letter, security, trust fund.

- Canada - New Brunswick: cash, negotiable bond, letter of credit, insurance bond.

- Índia: letter of credit, bond, trust fund, ou outros mecanismos aceitos pelas autoridades locais.

- Indonésia: term deposit, accounting reserve ou third party guarantee.

- Japão: cash, deposit guarantee e contribuições significativas para os casos da necessidade de tratamento de água.

- África do Sul: trust fund, cash deposit, letter of guarantee from a bank, ou outra forma de garantia aceita pelas autoridades locais.

- Suécia: bank guarantee, letters of credit, cash bonds.

- EUA - Arkansas: surety bonds, letter of credit.
- EUA - Nevada: bonds, letters of credit, trust fund, corporate guarantee of insurance.

- EUA - Texas: surety bonds, letter of credit, collateral bonds, escrow bonding, self-bonding, combined surety/escrow bonding.

Em 2005, o Diário Legislativo do Estado de Minas Gerais publicou o Projeto de Lei n. 2.194, encaminhado à Assembléia Legislativa pelo Governo do Estado, que dispõe sobre a concessão de garantias reais, no curso do licenciamento ambiental, pelos empreendimentos causadores de significativo dano ao meio ambiente. Nos termos do Projeto de Lei n. 2.194, o licenciamento ambiental de tais empreendimentos ficam sujeito à concessão, pelos empreendedores, de garantias reais para fazer face à recuperação de eventuais danos ambientais ou à implantação de medidas que os previnam (art. $1^{\circ}$ ) (Cruz, 2006). O Projeto de Lei n. 2.194 continua em trâmite na Assembléia Legislativa do Estado de Minas Gerais (ALMG, 2007).

Esse artigo apresenta os resultados da análise da aplicação de alguns instrumentos de garantia financeira na viabilidade de uma mina de grande porte no Estado de Minas Gerais.

\section{Metodologia}

O presente estudo é parte integrante da pesquisa desenvolvida no Programa de Pós-Graduação em Engenharia Geotécnica/UFOP, que resultou em uma dissertação de mestrado (Almeida, 2006). Para tal, foi realizado um levantamento bibliográfico com ênfase nos mecanismos de garantia financeira e consultas a professores, advogados, corretores de seguros, técnicos de órgãos reguladores da área de meio ambiente e empresas de mineração, para a obtenção de pontos de vista a respeito do fechamento de uma mina e verificação de como essa questão é tratada sob os aspectos econômico, ambiental e legal. A pesquisa foi desenvolvida com base em dados secundários disponibilizados na internet, em relatórios anuais de empresas de mineração e de órgãos reguladores, além de periódicos especializados na área de mineração e meio ambiente.

A delimitação da pesquisa compreendeu a análise do empreendimento Mina de Capão Xavier (CVRD), localizada no município de Nova Lima (MG), e a escolha do estudo de caso se deveu a fatores como:

- Porte do empreendimento.

- Plano de fechamento da mina, apresentado na fase de licenciamento ambiental aos órgãos reguladores.

- Polêmica a respeito dos problemas que poderiam ser ocasionados ao abastecimento de água da Região Metropolitana de Belo Horizonte (RMBH), devido ao rebaixamento do lençol freático, que resultou na abertura de uma CPI na Assembléia Legislativa de Minas Gerais (ALMG).

204 REM: R. Esc. Minas, Ouro Preto, 61(2): 203-209, abr. jun. 2008 
A avaliação do impacto dos mecanismos de garantia financeira na viabilidade da Mina Capão Xavier foi realizada com base nas informações contidas no PCA (ECOLAB 2003) e EIA (ECOLAB, 2002) do empreendimento, disponibilizado para consulta pública na FEAM e nas informações sobre a Mina de Capão Xavier (MBR, 2005) e (MBR, 2002), publicadas em periódicos especializados em mineração e disponibilizadas no sítio da MBR na internet.

\section{Resultados}

Para o estudo de caso e considerando-se o mercado brasileiro, foram avaliados seis cenários, adotando-se as seguintes premissas obtidas dos levantamentos bibliográficos realizados em Almeida (2006):

- Investimento total para implantação de Capão Xavier: US\$ 100 milhões.

- Investimento em instalações industriais - R\$ 84,4 milhões.

- Investimento inicial (acumulado até 2002) - R\$150,65 milhões (investimento total menos o investimento em instalações industriais).

- Cotação do dólar - R\$2,3505 (base 2001).

- Taxa mínima de atratividade do investimento/custo do capital $12 \%$ a.a.

- $2,89 \%$ do valor da receita do empreendimento custearão as despesas para a recuperação ambiental e fechamento da mina.

- Período de monitoramento e manutenção - 5 anos.

- Reembolso do valor provisionado e recuperado após os cinco anos da fase de monitoramento sem correção monetária, considerando-se que os rendimentos líquidos obtidos com o fundo serão revertidos para imprevistos e despesas correntes na fase de monitoramento e manutenções. A venda dos ativos (instalações industriais, máquinas, veículos e equipamentos) contribui, também, para o fundo, para custear imprevistos e despesas com monitoramento e manutenções.

Foram estudados seis cenários para análise da viabilidade, sendo:

- Cenário I - Considerou as despesas com recuperação ambiental e fechamento da mina diluída nas despesas diretas e sem provisionamento para o fechamento da mina.

- Cenário II - Considerou as despesas com recuperação ambiental e fechamento da mina, a serem efetivadas somente ao final da vida útil do empreendimento.

- Cenário III - Considerou as despesas com recuperação ambiental e fechamento da mina sem provisão para o fechamento da mina.

- Cenário IV - Inserção da garantia financeira na forma de um depósito mensal à vista em um fundo, no valor total de 2,829\% sobre a receita do empreendimento, para recuperação ambiental e fechamento da mina. Considerou-se a retirada cinco anos após o encerramento das operações. Considerou-se, também, o gasto anual com despesas para a recuperação ambiental e fechamento da mina.

- Cenário V - Inserção da garantia financeira na forma de uma apólice de seguro ou fiança bancária no valor total do custo de recuperação ambiental e fechamento da mina, renovável anualmente no valor total de $2,829 \%$ sobre a receita do empreendimento, para recuperação ambiental e fechamento. Foi considerado o custo da apólice de $4 \%$ para a renovação anual sobre o valor total de face da apólice. Gasto de 2,829\% sobre a receita anual com despesas para a recuperação ambiental e fechamento da mina.

- Cenário VI - Inserção da garantia financeira na forma de um depósito único à vista em um fundo de renda fixa, no valor total de 2,829\% sobre a receita do empreendimento, para recuperação ambiental e fechamento da mina. Considerouse a retirada cinco anos após o encerramento das operações. Gasto de 2,829\% sobre a receita anual com despesas para a recuperação ambiental e fechamento da mina.

A Tabela 1 apresenta um modelo consolidado das entradas de dados para o cálculo do cenário de I a VI, para o período de 2002 a 2028. As informações e cálculos detalhados são apresentados em Almeida (2006).

A Tabela 2 apresenta o resumo dos resultados para os cenários de I a VI.

A Figura 1 apresenta a evolução da Receita e do Fluxo de Caixa.

A Tabela 3 apresenta uma análise de sensibilidade simplificada que evidencia os efeitos da variação da taxa de atratividade do investimento/custo do capital sobre o Valor Presente Líquido(VPL).

A Figura 2 apresenta a evolução do VPL em função da variação da taxa de atratividade do investimento/custo do capital.

\section{Discussão}

Avaliando-se os cenários I a VI constata-se que o Cenário I (não pagamento de garantia financeira e as despesas com a recuperação ambiental foram consideradas nas despesas diretas), mantidas as premissas adotadas, é o mais satisfatório, em termos econômico-financeiros para a empresa de mineração, bem como aquele que oferece menor segurança para os órgãos reguladores e a sociedade. O Cenário VI oferece maior segurança para os órgãos reguladores e a sociedade, sendo o menos favorável em termos econômico-financeiros para a empresa de mineração. Os demais cenários (II a V) variam numa escala intermediária. 
Garantia financeira para fins de fechamento de mina e o seu impacto na viabilidade de uma mina..

Tabela 1 - Dados Consolidados (milhões R\$). Fonte: Almeida, 2006.

\begin{tabular}{|c|c|c|c|c|c|c|c|c|c|}
\hline \multirow{2}{*}{ Item } & \multicolumn{9}{|c|}{ Ano } \\
\hline & 2002 & 2003 & 2004 & 2005 & 2006 & 2007 & 2008 & 2009 & 2010 \\
\hline (1) Investimento Inicial & $-150,65$ & - & - & - & - & - & - & - & - \\
\hline (2) Investimento Instalações & - & $-6,90$ & $-1,50$ & $-30,40$ & $-31,90$ & $-0,60$ & $-13,10$ & - & - \\
\hline (3) Receita & - & 59,93 & 141,00 & 141,00 & 193,88 & 229,13 & 246,75 & 264,38 & 282,00 \\
\hline (4) Despesas Diretas & - & $-45,50$ & $-102,50$ & $-101,50$ & $-135,00$ & $-162,40$ & $-175,70$ & $-188,90$ & $-203,20$ \\
\hline (5) Despesas com recuperação ambiental & - & $-1,70$ & $-3,99$ & $-3,99$ & $-5,48$ & $-6,48$ & $-6,98$ & $-7,48$ & $-7,98$ \\
\hline (6.1) Cenário I - Sem provisionamento & - & - & - & - & - & - & - & - & - \\
\hline (6.2) Cenário II - Desembolso último ano do projeto. & - & - & - & - & - & - & - & - & - \\
\hline (6.3) Cenário III - Despesas com recuperação Ambiental & - & $-1,70$ & $-3,99$ & $-3,99$ & $-5,48$ & $-6,48$ & $-6,98$ & $-7,48$ & $-7,98$ \\
\hline (6.4) Cenário IV - Provisionamento mensal & - & $-1,70$ & $-3,99$ & $-3,99$ & $-5,48$ & $-6,48$ & $-6,98$ & $-7,48$ & $-7,98$ \\
\hline $\begin{array}{l}\text { (6.5) Cenário V - Despesas com apólice de seguro ou } \\
\text { fiança bancária }\end{array}$ & - & $-4,71$ & $-4,71$ & $-4,71$ & $-4,71$ & $-4,71$ & $-4,71$ & $-4,71$ & $-4,71$ \\
\hline (6.6) Cenário VI - Depósito inicial único & - & $-117,89$ & - & - & - & - & - & - & - \\
\hline (7.1) Fluxo de Caixa - Cenário I $\Sigma(1$ a $4+6.1)$ & $-150,65$ & 7,53 & 37,00 & 9,10 & 26,98 & 66,13 & 57,95 & 75,48 & 78,80 \\
\hline (7.2) Fluxo de Caixa - Cenário II $\Sigma(1$ a $4+6.2)$ & $-150,65$ & 7,53 & 37,00 & 9,10 & 26,98 & 66,13 & 57,95 & 75,48 & 78,80 \\
\hline (7.3) Fluxo de Caixa - Cenário III $\Sigma(1$ a $4+6.3)$ & $-150,65$ & 5,83 & 33,01 & 5,11 & 21,50 & 59,65 & 50,97 & 68,00 & 70,82 \\
\hline 7.4) Fluxo de Caixa - Cenário IV $\Sigma(1$ a $5+6.4)$ & $-150,65$ & 4,13 & 29,02 & 1,12 & 16,02 & 53,17 & 43,99 & 60,52 & 62,84 \\
\hline (7.5) Fluxo de Caixa - Cenário V $\Sigma(1$ a $5+6.5)$ & $-150,65$ & 1,12 & 28,30 & 0,40 & 16,79 & 54,94 & 46,26 & 63,29 & 66,11 \\
\hline (7.6) Fluxo de Caixa - Cenário VI $\Sigma(1$ a $5+6.6)$ & $-150,65$ & $-112,06$ & 33,01 & 5,11 & 21,50 & 59,65 & 50,97 & 68,00 & 70,82 \\
\hline \multirow{2}{*}{ Item } & \multicolumn{9}{|c|}{ Ano } \\
\hline & 2011 & 2012 & 2013 & 2014 & 2015 & 2016 & 2017 & 2018 & 2019 \\
\hline (1) Investimento Inicial & - & - & - & - & - & - & - & - & - \\
\hline (2) Investimento Instalações & - & - & - & - & - & - & - & - & - \\
\hline (3) Receita & 282,00 & 282,00 & 282,00 & 282,00 & 282,00 & 282,00 & 282,00 & 282,00 & 211,50 \\
\hline (4) Despesas Diretas & \begin{tabular}{|l|}
$-199,70$ \\
\end{tabular} & $-197,60$ & $-192,10$ & $-193,30$ & $-193,60$ & $-195,10$ & $-195,60$ & $-202,70$ & $-157,80$ \\
\hline (5) Despesas com recuperação ambiental & $-7,98$ & $-7,98$ & $-7,98$ & $-7,98$ & $-7,98$ & $-7,98$ & $-7,98$ & $-7,98$ & $-5,98$ \\
\hline (6.1) Cenário I - Sem provisionamento & - & - & - & - & - & - & - & - & - \\
\hline (6.2) Cenário II - Desembolso último ano do projeto. & - & - & - & - & - & - & - & - & - \\
\hline (6.3) Cenário III - Despesas com recuperação Ambiental & $-7,98$ & $-7,98$ & $-7,98$ & $-7,98$ & $-7,98$ & $-7,98$ & $-7,98$ & $-7,98$ & $-5,98$ \\
\hline (6.4) Cenário IV - Provisionamento mensal & $-7,98$ & $-7,98$ & $-7,98$ & $-7,98$ & $-7,98$ & $-7,98$ & $-7,98$ & $-7,98$ & $-5,98$ \\
\hline $\begin{array}{l}\text { (6.5)Cenário V - Despesas com apólice de seguro ou } \\
\text { fiança bancária }\end{array}$ & $-4,71$ & $-4,71$ & $-4,71$ & $-4,71$ & $-4,71$ & $-4,71$ & $-4,71$ & $-4,71$ & $-4,71$ \\
\hline (6.6)Cenário VI - Depósito inicial único & - & - & - & - & - & - & - & - & - \\
\hline (7.1) Fluxo de Caixa - Cenário I $\Sigma(1$ a $4+6.1)$ & 82,30 & 84,40 & 89,90 & 88,70 & 88,40 & 86,90 & 86,40 & 79,30 & 53,70 \\
\hline (7.2) Fluxo de Caixa - Cenário II $\Sigma(1$ a $4+6.2)$ & 82,30 & 84,40 & 89,90 & 88,70 & 88,40 & 86,90 & 86,40 & 79,30 & 53,70 \\
\hline (7.3) Fluxo de Caixa - Cenário III $\Sigma(1$ a $4+6.3)$ & 74,32 & 76,42 & 81,92 & 80,72 & 80,42 & 78,92 & 78,42 & 71,32 & 47,72 \\
\hline 7.4) Fluxo de Caixa - Cenário IV $\Sigma(1$ a $5+6.4)$ & 66,34 & 68,44 & 73,94 & 72,74 & 72,44 & 70,94 & 70,44 & 63,34 & 41,74 \\
\hline (7.5) Fluxo de Caixa - Cenário V $\Sigma(1$ a $5+6.5)$ & 69,61 & 71,71 & 77,21 & 76,01 & 75,71 & 74,21 & 73,71 & 66,61 & 43,01 \\
\hline (7.6) Fluxo de Caixa - Cenário VI $\Sigma(1$ a $5+6.6)$ & 74,32 & 76,42 & 81,92 & 80,72 & 80,42 & 78,92 & 78,42 & 71,32 & 47,72 \\
\hline \multirow{2}{*}{ Item } & \multicolumn{9}{|c|}{ Ano } \\
\hline & 2020 & 2021 & 2022 & 2023 & 2024 & 2025 & 2026 & 2027 & 2028 \\
\hline (1) Investimento Inicial & - & - & - & - & - & - & - & - & - \\
\hline (2) Investimento Instalações & - & - & - & - & - & - & - & - & - \\
\hline (3) Receita & 141,00 & - & - & - & - & - & - & - & - \\
\hline (4) Despesas Diretas & \begin{tabular}{|l|}
$-101,70$ \\
\end{tabular} & $-2,50$ & - & - & - & - & - & - & - \\
\hline (5) Despesas com recuperação ambiental & $-3,99$ & - & - & - & - & - & - & - & - \\
\hline (6.1) Cenário I-Sem provisionamento & - & - & - & - & - & - & - & - & - \\
\hline (6.2) Cenário II - Desembolso último ano do projeto. & - & $-117,89$ & - & - & - & - & - & - & - \\
\hline (6.3) Cenário III - Despesas com recuperação Ambiental & $-3,99$ & - & - & - & - & - & - & - & - \\
\hline (6.4) Cenário IV - Provisionamento mensal & $-3,99$ & - & - & - & - & - & 117,89 & - & - \\
\hline $\begin{array}{l}\text { (6.5) Cenário } \mathbf{V} \text { - Despesas com apólice de seguro ou } \\
\text { fiança bancária }\end{array}$ & $-4,71$ & - & - & - & - & - & - & - & - \\
\hline (6.6)Cenário VI - Depósito inicial único & - & - & - & - & - & - & - & - & - \\
\hline (7.1) Fluxo de Caixa - Cenário I $\Sigma(1$ a $4+6.1)$ & 39,30 & $-2,50$ & - & - & - & - & - & - & - \\
\hline (7.2) Fluxo de Caixa - Cenário II $\Sigma(1$ a $4+6.2)$ & 39,30 & $-120,39$ & - & - & - & - & - & - & - \\
\hline (7.3) Fluxo de Caixa - Cenário III $\Sigma(1$ a $4+6.3)$ & 35,31 & $-2,50$ & - & - & - & - & - & - & - \\
\hline 7.4) Fluxo de Caixa - Cenário IV $\Sigma(1$ a $5+6.4)$ & 31,32 & $-2,50$ & - & - & - & - & 117,89 & - & - \\
\hline (7.5) Fluxo de Caixa - Cenário V $\Sigma(1$ a 5+6.5) & 30,60 & $-2,50$ & - & - & - & - & - & - & - \\
\hline (7.6) Fluxo de Caixa - Cenário VI $\Sigma(1$ a $5+6.6)$ & 35,31 & $-2,50$ & - & - & - & - & 117,89 & - & - \\
\hline
\end{tabular}

206 REM: R. Esc. Minas, Ouro Preto, 61(2): 203-209, abr. jun. 2008 
Maurício Rios de Almeida et al.

Tabela 2 - Resumo dos resultados obtidos de VPL e TIR. Fonte: Almeida, 2006.

\begin{tabular}{c|c|c}
\hline Cenário & TIR (\%) & VPL (R\$ milhões) \\
\hline I & $26,24 \%$ & 226,91 \\
\hline II & $26,06 \%$ & 213,22 \\
\hline III & $23,88 \%$ & 184,67 \\
\hline IV & $21,56 \%$ & 151,13 \\
\hline V & $21,66 \%$ & 150,52 \\
\hline VI & $16,15 \%$ & 91,63 \\
\hline
\end{tabular}

Entretanto é preciso que o empresário fique atento quanto ao Cenário I, devido às incertezas advindas do longo tempo de maturação, desenvolvimento e fechamento de uma mina, uma vez que podem ocorrer desvios e imprevistos nas avaliações desenvolvidas na fase de viabilidade do empreendimento, obrigando, no futuro, caso as previsões tenham sido subavaliadas ou não refletirem o planejamento inicial, o aporte de mais recursos que pode ocorrer numa fase

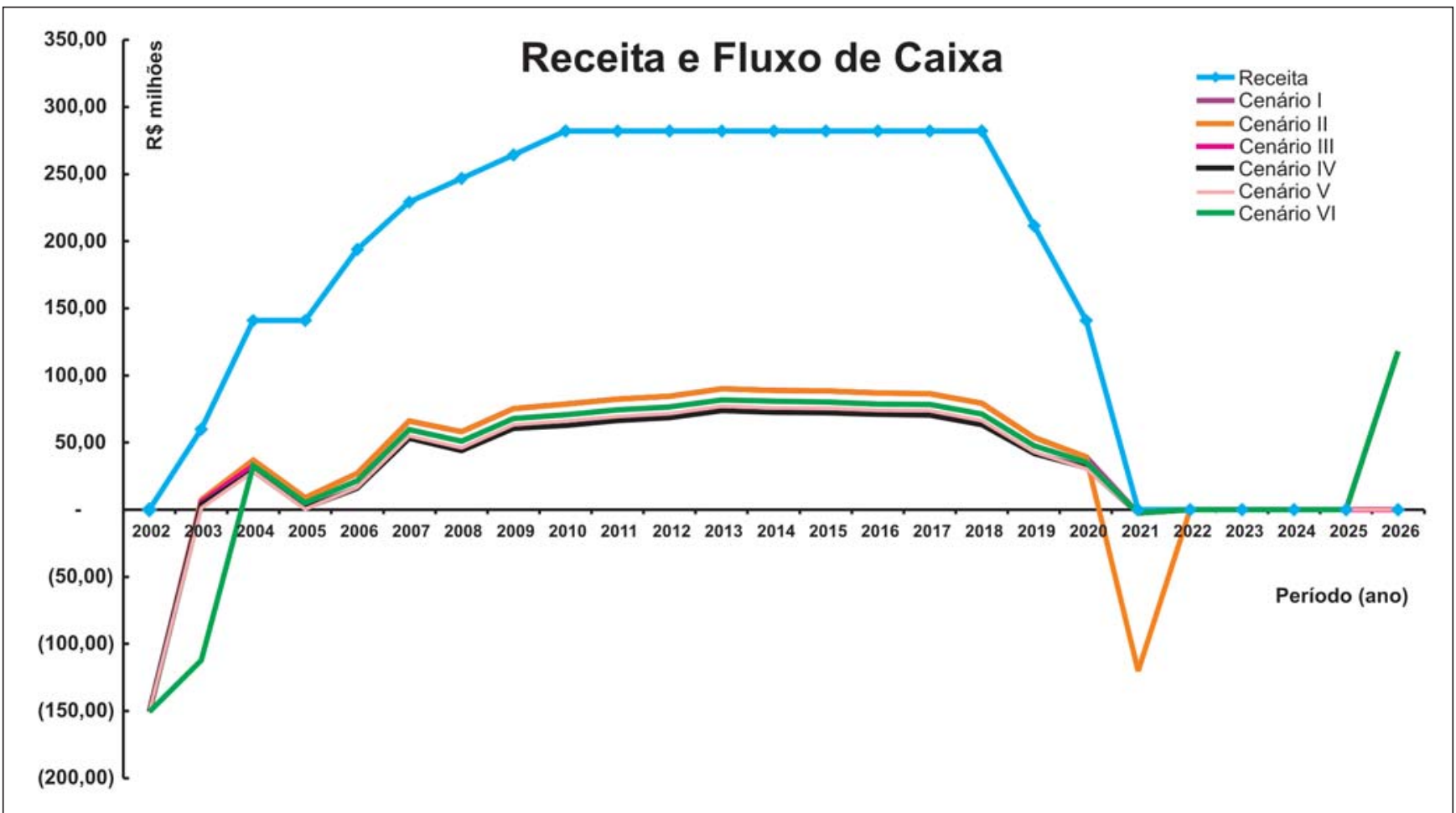

Figura 1 - Evolução da Receita e Fluxo de Caixa. Fonte: Almeida, 2006.

Tabela 3 - Análise de sensibilidade.

\begin{tabular}{c|c|c|c|c|c|c|c|c}
\hline \multicolumn{1}{c}{ Variação do Custo do Capital (\%) X VPL (R\$ milhões) } \\
\hline Cenário & $\mathbf{6 \%}$ & $\mathbf{8 \%}$ & $\mathbf{1 0 \%}$ & $\mathbf{1 2 \%}$ & $\mathbf{1 5 \%}$ & $\mathbf{2 0 \%}$ & $\mathbf{2 5 \%}$ & $\mathbf{3 0 \%}$ \\
\hline I & 475,62 & 373,53 & 292,24 & 226,91 & 151,24 & 65,35 & 10,44 & $-26,20$ \\
\hline II & 436,65 & 346,21 & 272,96 & 213,22 & 142,96 & 61,66 & 8,74 & $-27,01$ \\
\hline III & 408,32 & 316,46 & 243,37 & 184,67 & 116,75 & 39,78 & $-9,29$ & $-41,94$ \\
\hline IV & 371,88 & 272,32 & 207,66 & 151,13 & 86,99 & 16,00 & $-28,32$ & $-57,38$ \\
\hline V & 357,32 & 272,32 & 204,74 & 150,52 & 87,88 & 17,12 & $-27,79$ & $-57,50$ \\
\hline VI & 333,86 & 232,60 & 153,72 & 91,63 & 21,44 & $-55,39$ & $-102,24$ & $-132,00$ \\
\hline
\end{tabular}




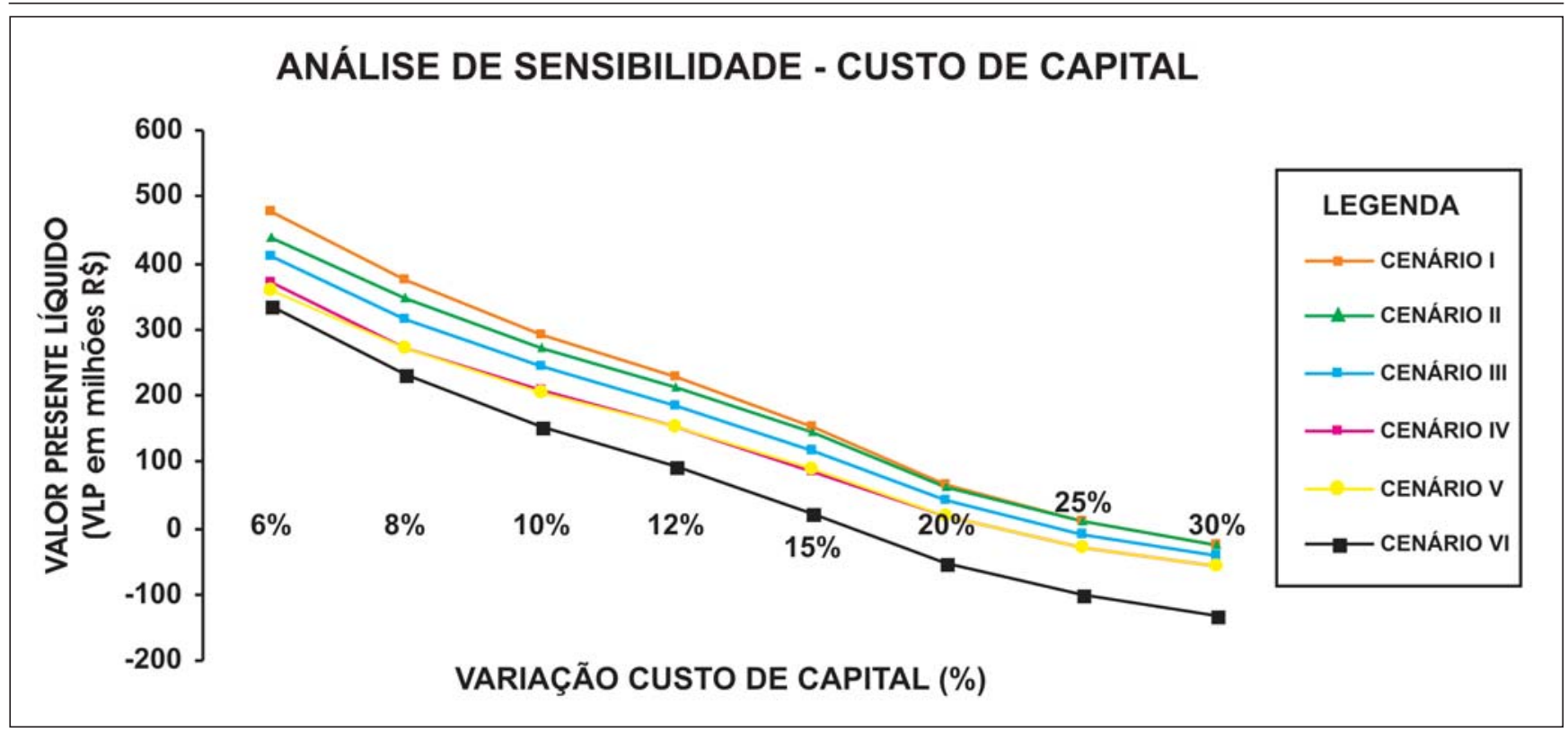

Figura 2 - Análise de sensibilidade da variação do custo de capital.

onde não haverá mais faturamento/receita, podendo impactar fortemente a saúde financeira da empresa e, num cenário mais sombrio, havendo a falta do operador, externalizar os custos da recuperação e fechamento para os órgãos reguladores e para sociedade.

Por outro lado, quando se fala de pagamento da garantia financeira, há que se levar em conta que isso significa a retenção ou retirada de recursos financeiros do resultado da empresa, que poderiam ser aplicados nas operações, investimentos, pesquisas, etc., com "redução" da competitividade da empresa. Os cenários III a VI apresentam os impactos dos mecanismos de garantia financeira (com desembolso de recursos) na viabilidade do empreendimento. $\mathrm{O}$ cenário II vem demonstrar o que aborda a literatura a respeito do aspecto de que, em geral, custos de fechamento baseado na avaliação do Valor Presente Líquido (VPL), após a fase de produção, não afetam o VPL, portanto, a decisão de investimento. Neste caso, podem ser avaliados e comparados os resultados dos Cenários I e II.

Lima et al. (2003) e Lima (2005) mostram que a avaliação de um projeto é baseada no Valor Presente Líquido (VPL). Em geral, custos de fechamento, depois da vida de um projeto (tipicamente de- pois de 20 anos), não afetarão o VPL e, portanto, a decisão de investimento. Uma empresa de mineração pode, então, comprometer-se aos altos custos de fechamento sem que isto incorra em impacto na decisão de investimento. Entretanto o fluxo de caixa exigido para implementar o plano de fechamento pode, em alguns casos, ser tão alto que comprometa a viabilidade da empresa quando do fechamento.

\section{Conclusão}

Existem vantagens e desvantagens em cada mecanismo de garantia financeira e os órgãos reguladores deverão adotar, após amplo debate com as partes envolvidas (empresários e sociedade), a que melhor se adapta à realidade de cada mina. Deve-se, ter como base, o porte da empresa e do empreendimento de mineração, proporcionando o maior número de alternativas pela legislação e facultando ao empresário a escolha que melhor atenda aos seus interesses, sem, contudo, impor riscos desnecessários à sociedade.

É importante que os órgãos reguladores e as empresas de mineração cheguem a um acordo a respeito dos padrões esperados de reabilitação, o valor e ins- trumentos adequados de garantia financeira, antes que o projeto de mineração seja comprometido. Essas providências devem fazer parte do acordo ou licença sob a qual o desenvolvimento da mineração procede. O valor da garantia pode ser ajustado após o requerimento, mas o processo de ajuste deve ser bem entendido e estar de acordo com o avanço da reabilitação, evitando desagradáveis surpresas para as partes envolvidas (Miller, 2005).

A respeito dos resultados obtidos na pesquisa e com base em todas as informações, avaliações e cálculos realizados para o estudo de caso da Mina de Capão Xavier, conclui-se que, para os cenários de I a VI avaliados, o empreendimento mantém a sua viabilidade, considerando-se às premissas adotadas.

\section{Referências bibliográficas}

ASSEMBLÉIA LEGISLATIVA DE MINAS GERAIS - ALMG Projeto de Lei 2194/05. Disciplina a concessão de garantias reais a serem apresentadas no curso do licenciamento ambiental, por empreendimentos potencialmente causadores de dano significativo ao meio ambiente. 2007. Disponível em: $<$ http://www.almg.gov.br $>$. Acesso em: 03 novembro. 2007. 
Maurício Rios de Almeida et al.

ALMEIDA, M. R. Avaliação dos mecanismos de garantia financeira para fins de fechamento de mina $e$ o seu impacto na viabilidade de projeto de mineração de grande porte no estado de Minas Gerais. Ouro Preto: Universidade Federal de Ouro Preto, 2006. 189f. (Dissertação de Mestrado).

BAMBURRA, E. V. Garantias financeiras e o fechamento de mina. In: SEMINÁRIO BRASIL \& CANADÁ DE RECUPERAÇÃO AMBIENTAL DE ÁREAS MINERADAS, Florianópolis, 2003.

CRUZ, J. C. C. Fechamento de mina: aspectos técnicos, jurídicos e socioambientais. Campinas: UNICAMP, 2006. 309 f. (Tese de Doutorado em Geociências).

ECOLAB Plano de Controle Ambiental. Programa de Produção Mineral.
RT-MBR-311/03. Projeto Capão Xavier. 2003. Disponível para consulta: FEAM. Acesso em 2005 e 2006.

ECOLAB Estudo de Impacto Ambiental.Projeto Capão Xavier. RT.287/02. v. I e II. 2002. Disponível para consulta: FEAM. Acesso em 2005 e 2006.

LIMA, H. M., COSTA, F. L., CALDEIRA, V., PEIXOTO, R. J. Financial guarantee for mine closure. Rem - Revista Escola de Minas, v. 56, n. 3, p. 181-186, 2003.

LIMA, H. M. Aspectos técnicos y metodologicos del cierre de minas. In: Roberto Blanco Torrens; Paúl Carrión Mero. (Org.). Ordenamiento Y Cierre de Minas. Guayaquil: Imprenta Mistral, 2005. v. Tomo II, p. 51-92.

MILLER, G. C. Financial assurance for mine closure and reclamation (Report). International Council on Metals and the Environment (ICME): Disponível em <http://www.icmm.com>. 2005.

MINERAÇÕES BRASILEIRAS REUNIDAS S.A. - MBR. O Projeto Capão Xavier. 2005. Disponível em <http://www.mbr.com.br/capao/index.html>. Acesso em 07 de setembro de 2005.

MINERAÇÕES BRASILEIRAS REUNIDAS S.A. - MBR. Relatório Anual. 2002. < <http:// www.mbr.com.br/inf_financeiras/inf_financeiras.asp>. Acesso em 29 de setembro de 2005.

REIS, N. L., BARRETO, M. L. Desativação de empreendimento mineiro no Brasil. São Paulo: Signus Editora, 2000. 48 p.

Artigo recebido em 25/06/2007 e aprovado em 01/12/2007.

\section{Você não vai esconder seu trabalho de anos de pesquisas numa revista que nunca se sabe quando vai ser publicada, ou que ninguém lê... Vai?}

\section{Conheça e Publique-o na REM 72 anos divulgando ciência.}

$* * * * *$

\section{www.rem.com.br}

